\title{
Mobile Ad Hoc Networking Approach to Detecting and Querying Events Related to Farm Animals
}

\author{
Milena Radenkovic Bartosz Wietrzyk \\ School of Computer Science and IT \\ University of Nottingham \\ Nottingham, NG8 1BB, UK \\ $\{$ mvr, bzw $\} @$ cs.nott.ac.uk
}

\begin{abstract}
This paper proposes a mobile ad hoc networking approach to detecting and querying events related to farm animals such as oestrus, animal diseases and decreased efficiency of pastures. This problem is essential because inattention to the health or welfare of the animals can lead to reduced productivity and death of valuable stock.

The proposed approach guided by the identified requirements utilizes the available networking infrastructure but also works in a fully ad hoc infrastructure-less conditions. Data is acquired from the animal mounted devices using reliable multi hop wireless communication. Data can be also accessed from a portable device directly connecting to the sensor devices over wireless communication. We perform a simulation based evaluation of our approach showing that the proposed acquisition of data from the animal mounted devices is feasible even using energy efficient, low range, low bandwidth wireless communication.
\end{abstract}

\section{Introduction}

The production of beef and milk has always been a major constituent of agriculture in the United Kingdom. The total number of cattle in England in 2003 was estimated to be over 5,709,000. The beef and veal consumption in 2002 was on average $19.7 \mathrm{~kg}$ per capita in UK and $19.6 \mathrm{~kg}$ in EU [1].

There has always been a need for livestock producers to be able to 'observe' their animals as often as possible. Inattention to the wellbeing of the animals, whether it be a health or welfare issue can lead to reduced productivity and death of valuable stock [2]. The supervision of reproductive processes such as pregnancy and oestrus is essential for breeding the animals in particular when the modern farming technologies such as artificial insemination are applied [3]. It is often the case that the farmer has neither time nor resources to 'see' the animals regularly and even when she does, she may not be in a position to identify reproductive state of the individuals or some of their more deep-rooted health problems. The ability to be informed of changes of the animals' health, reproductive status and welfare can reduce the current reliance on manpower and improve the decision making process.

The contribution of this paper is an ad hoc networking approach to detecting and querying events related to farm animals such as oestrus, animal diseases or decreased efficiency of pastures. This work is informed by experiences from our previous research in nationwide cattle monitoring system [4]. We identify the requirements and challenges for the event detection system and propose an approach that addresses them. It utilizes available infrastructure and can also work in fully ad hoc infrastructure-less conditions. We then evaluate our approach over an extensive simulation.

The paper is organized in the following way. Section 2 defines the requirements for the event detection system. Section 3 describes the challenges. Section 4 briefly reports on the existing approaches. Section 5 proposes our approach. Section 6 describes simulation based evaluation of the proposed approach.

\section{Requirements}

The cattle production in UK is performed by private enterprises. Each of them can manage several pastures and farm buildings where the animals are kept. Cattle are kept in UK for production of milk and beef. Milk is produced by dairy herds, which also produce calves of dairy cattle. The structure of the beef production enterprises is more complex. The calves are produced 
by suckler herds and then sold to various enterprises often specializing in cattle of specific age [1]. The average number of cattle held by a British enterprise in 1998 was 72 animals [5]. This average is higher for some types of enterprises e.g. calf-based semi intensive finishers had in 2003147 animals on average [1].

The cattle can be kept all the year on the pastures or all the year in the farm buildings but the most common practice is to keep them on the pastures in the warmer half of the year and indoors in the other [5]. Depending on the conditions stocking density of the pastures can range from 2 to 7 animals per ha [5]. In the simplest grazing system (continuous grazing), cattle are allowed to graze freely on the whole available pasture. All the other systems constrain area available to the animals [5]. The dairy cows are milked twice or thrice each day in most cases with the involvement of people. Beef cattle is handled far less frequently and therefore are generally more fearful of human intervention [6].

There are four major reasons for cattle monitoring: (1) detecting oestrus [2, 7-12], (2) detecting animal diseases [2, 7-12], (3) assessing efficiency of pastures and paddocks [2]. Oestrus detection is highly important in case of artificial insemination (AI), which is currently a common practice in case of dairy cows and is being introduced for suckler cows [3]. Oestrus detection can be performed according to several factors but relying on too few can lead to a high number of false positive errors $[10,11]$. These factors can be divided into behavioral and milk parameters. The latter include temperature and amount of progesterone [11, 12]. They can be measured by automatic milking facilities but in case of progesterone it is still challenging [12]. The behavioral factors include intensity of walking, food intake and acceptance of mounting from males and females i.e. 'standing-to-bemounted' (STMB). Walking activity can be measured by a pedometer mounted on an animal's leg. The highest probability of conception takes place between 6 to 24 hours after the detected increase in walking activity [9]. Food intake can be measured by an accelerometer mounted on animal's neck or various sensors inside the rumen [2]. Detecting STMB is so far most challenging. The current approaches [11] include painting animal's rump, gluing a simple device to animal's rump or using detector animals (a surgically modified bull, an androgenised steer or cow) with marking devices. None of these approaches gives satisfactory results.

Another reason for monitoring farm animals is detecting diseases such as mastitis and other infection diseases, metabolic diseases and lameness. Similarly to oestrus, diseases can be detected using milk and/or behavior monitoring. The appropriate milk parameters include temperature and conductivity, which are relatively easy to measure by automatic milking facilities. Behavioral factors relevant for disease monitoring include intensity of walking and food intake. They can be monitored using similar methods as in case of oestrus [11].

Efficiency of pastures and paddocks can be estimated on the base of feed intake [2], which can be measured by a pedometer or an accelerometer mounted on the animal's neck. This information can be useful in taking decisions concerning moving animals to a different pasture or from a pasture to a farm building. This information can also help in planning purchases or sales of the pastureland.

In this paper we will concentrate on monitoring suckler herds. Contrary to the dairy herds, suckler herds are far less handled by humans [6], which justifies use of modern wireless and wired networking. Because of their reproductive character suckler herds require much more attention in terms of monitoring and handling than herds managed by finishers or store producers $[3,6]$. We will concentrate on monitoring walking and feed intake intensity of cattle in order to detect oestrus, pregnancy and animal diseases such as mastitis or lameness.

A potential animal monitoring system would notify a farmer or a stockman about an event such as disease or lack of feed on a pasture using GSM text messages, emails or notice boards. A user (i.e. a farmer, a stockman or a veterinary) should be able to query all the currently ill animals or animals which are or soon going to be ready for insemination. It should be possible for a user to retrieve data about a certain animal such as current and past diseases, oestruses and pregnancies. A more advanced user such as a veterinary or a skilled stockman could be able to see a graph of a particular factor (e.g. intensity of walking or feed intake) together with its deviation from the average, referring to a particular animal. Querying should be possible from the farm terminals, remotely over Internet, from mobile phones, home and office PCs. Both input data and detected events should be retained as long as the animal it concerns stays on the farmer's hold.

Earlier selling and purchasing of livestock took place in the auctions involving presence of the animals. Recently the transport of the animals is tried to be limited as it results in stress degrading the quality of meat. Because of that, Internet auctions are being introduced [5]. It is beneficial to provide potential buyers with the selected data from the envisaged system so they can evaluate the animals before the purchase. 


\section{Challenges}

In this section, we identify the challenges for the cattle monitoring system. The major challenges include:

Functionality including scope of detected events and monitored parameters. Functionality also refers to availability of real-time and historical input data (measurements), output data (results of processing) and detected user definable events.

Decreased maintenance and deployment costs - the costs of the system should be kept low. This includes costs of hardware, involvement of workforce in maintenance and deployment, particularly the workforce with certain technical skills and costs of the third party communication services such as GPRS connectivity.

Spatial distribution of farming infrastructure pastures belonging to a farm can be located far from each other and far from the farm buildings. That can make it difficult to access from the farm building data collected by the animal mounted devices.

Limited availability of the networking infrastructure - the networking infrastructure available on farms can be very limited. It may not be possible to connect the pastures to the farm buildings with the wired network due to their spatial distance or economic constrains. Utilization of the GSM telephony for such connectivity may also be impossible because of lack of GSM coverage or economic constrains. The availability of the Internet connection in the farm buildings can also be limited.

Increased Reliability - this refers to probability of successful retrieval of measurements from the animal mounted devices but also false positive detections of the monitored events.

Impact on the animals - The animal mounted devices should not cause any excessive annoyance to the animals in terms of their construction, weight and placement [13]. Otherwise the animals will be given an incentive to destroy the devices.

Security - access to the collected data should be restricted and sufficiently secured as this data can be business sensitive.

\section{Related work}

This section evaluates current approaches to the animal monitoring. There is a number of existing approaches concerning animal monitoring. None of them satisfies our requirements but we briefly evaluate them in order to better understand our challenges.

Stationary sensor networks for animal monitoring that are typically deployed today have small to medium scale (tens to hundreds of sensors), span small to medium geographical distances (tens to hundreds of square miles) and are short to medium term (hours to months). The sensor data is typically archived in a powerful server geographically collocated with the sensors that is usually fully replicated on the predetermined powerful servers in the labs e.g. The Great Duck Island [14].

RFIDs are an established approach to tracking domestic animals [7]. They allow reading an animal identifier by a fixed reader that provides information that a given animal has been present at certain time at a given location. The advantages of this approach include: (1) low price, (2) small size - can be mounted in an animal as an implant, (3) long life due to lack of need for energy supply. The disadvantages include limited scope of provided information and unreliable RFID readings (especially in cases when animals move too fast or many of them are present near the reader).

Archival tags are monitoring devices that can be fitted on animals, can store relevant measurements for an animal and can be read by a handheld device [15]. This is the most reliable approach but expensive in terms of maintenance cost because the animals must be individually approached by stockmen. The scope of monitored factors is potentially large. The cost of deployment is high due to the price of monitoring devices.

In the case of radio tags, the measurements from monitoring devices mounted on animals can be read over wireless communication. This approach is often used in wildlife monitoring [13]. The ready made collars and receivers are made by various companies [16-20] and available off the shelf. The important limitation of this approach is that the receiver's aerial must be mounted in the place visited by all the animals. Finding such a place can be difficult in case of beef cattle fed by continuous grazing, i.e. roaming on a large meadow without interacting with humans. Then the data from the receiver must be transported to a server, which is not always an easy to automate task and may cause high maintenance costs.

Animal GSM telemetry. Another approach to retrieving data from the animal mounted devices utilizes GSM telephony [2]. Such collars are already available on the market [20]. In case of monitoring of large number of animals this approach can be financially challenging due to the cost of GSM communication, i.e. the maintenance costs. Another disadvantage is that GSM transceivers consume large amounts of energy. That can potentially lead to a considerable effort necessary to replace the batteries every few days. These limitations were very briefly addressed [2] by putting GSM, in particular GPRS 
transceivers only on a subset of animals. The devices without GPRS transceivers transfer their measurements through the GPRS-enabled devices.

Store and forward approach that was used in ZebraNet project [21] for monitoring zebras at the Mpala Research Centre in Kenya can also be used. In ZebraNet, devices mounted on zebras were transferring all their measurements to all other devices in their range. This approach was not scalable due to limited storage space of the devices and was feasible only because of the small number of monitored animals. The retrieval of the aggregated measurements from animals required approaching them by humans, which increased the maintenance costs.

There has been research done in controlling animals [22], which involved mounting devices on animals. These devices were producing sounds whenever an animal tried to leave a virtual paddock. The referenced paper concentrates on the automatic control aspect of the proposed application rather than on the utilized wireless communication.

\section{Proposed approach}

This section presents the proposed approach to the cattle monitoring system, which is shown in Figure 1. An animal mounted device has a form of a collar with the built in accelerometer measuring intensity of feed intake [2]. The walking intensity is measured by the pedometer mounted on the animal's leg [2, 7-12]. In both cases the impulses are counted for two hours [9]. The measurements from the pedometer are acquired by the collar over the wireless communication. The measurements from the pedometer and accelerometer are stored and processed by the collar every two or at most four hours in order to detect oestrus, pregnancy and animal diseases.

The collars have wireless network interfaces and transmit input data (i.e. measurements) and output data (e.g. detected diseases, oestrus) to the farm servers over the gateways. The farm servers store the real time and historic data. These servers also detect user defined events and issue notifications to users' mobile phones or message boards mounted in farm buildings or offices. These events are defined as logical functions of the output data calculated by the collars, e.g. 'The cow $\mathrm{X}$ on the pasture $\mathrm{Y}$ will be ready for insemination in 4 hours' or 'The paddock Z occupied currently by animals have efficiency below $10 \%$.

The users can query the data stored on servers including input and output data either locally on the farm or remotely over Internet using a PC at home or office or a WAP enabled mobile phone. The selective access to the data stored on the farm servers can be provided to business counterparts to give them the opportunity to evaluate the animals before the purchase.

In the envisaged system animal mounted collars and optionally stationary gateways are powered by batteries recharged by solar cells similarly as in ZebraNet [21]. Leg mounted pedometers use an internal battery for measurement and storage of data together with an external radiating power source mounted in the collar for transmitting data [8].

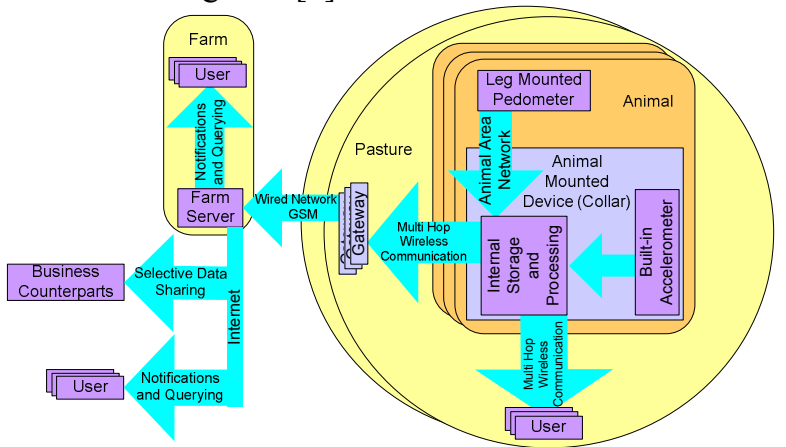

Figure 1. Diagram of the proposed approach

The example deployment of the system is shown in Figure 2. The data is regularly transferred from the animal mounted nodes to the gateways over multi hop communication, if the gateways are deployed. In multi hop communication every node participates in routing and forwarding of the packets. The multi hop path to a gateway can become unavailable when the topology splits into separated islands of connectivity (partitions). In that case a node retries after a given period of time (e.g. one minute) trying different gateways when more than one is deployed. We aim to achieve reliability in transferring data to the gateways by (re)sending it until receiving the acknowledgement from a gateway.

Where possible the gateways can be connected to farm servers over a wired network connection as in Pasture 1 and Pasture 2. If a pasture is located far from farm buildings it is possible to use GSM telephony to connect pastures to farm servers as in Pasture 3.

A user located in close proximity to the pasture is provided with the best effort access to the input and output data stored on the animal mounted devices. Therefore, users will be able to query the data from a PDA or a smart phone connecting directly to the animal mounted devices over the multi hop wireless communication. To address partitioning, our approach of self organized, network aware caching [4] can be utilized. Queries can be issued by flooding the network. All the devices knowing a complete or partial answer to the query from data they collected or cache would send a reply to the querying users. If a gateway is within the same island of connectivity as the user's device it can 
fetch the answer to the query directly from the farm server.

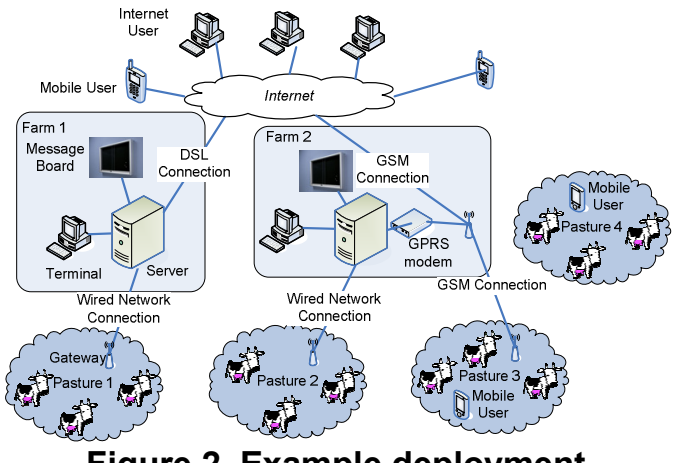

Figure 2. Example deployment

\section{Simulation}

\subsection{Methodology}

This section describes how we evaluate the proposed approach. We simulate different number of nodes with the maximal and minimal realistic stocking densities (2 animals/ha and 7 animal/ha [5]) for the duration of 2 simulated hours. We use the established network simulator ns-2 [23]. Nodes move with the speed of $0.6 \mathrm{~m} / \mathrm{s}$ (preferred walking speed for cattle [24]) according to the Random Waypoint Model [25] making stops from zero to one minute. They aim at sending (once) $16 \mathrm{~B}$ of data to a gateway using AODV [26] multi hop wireless routing protocol. If the nodes cannot contact a gateway, they wait one minute and try again. We compared the approaches with one stationary gateway and one animal mounted gateway. The stationary gateway is mounted in the middle of one of the sides of the simulation area. We use a low range wireless protocol (IEEE 802.15.4) assuming 10m range of transmitters and a longer range protocol (IEEE $802.11 \mathrm{~b}$ ) assuming $250 \mathrm{~m}$ range of transmitters. We measure success ratio i.e. the percentage of nodes that successfully sent the measurements to the gateways within 2 hours and network overhead i.e. the total amount of transmitted data.

\subsection{Simulation results}

Figure 3a shows the influence of number of network nodes on the success ratio (SR) in conditions of highest possible stocking density. We can see that in case of long-range communication (IEEE 802.11b) number of nodes and type of gateways (stationary or mobile, i.e. animal mounted) have little influence - the SR stays above $97.7 \%$. In case of short range communication (IEEE 802.15.4), success ratio falls close to $90 \%$ in case of a stationary gateway but is always above $95 \%$ for a mobile gateway. This is because the mobile gateway changing its position comes to a close proximity with larger number of nodes.

Figure $3 \mathrm{~b}$ shows the influence of number of nodes on network overhead (NO) in conditions of highest possible stocking density. In all examined cases, it grows almost linearly with the number of nodes. In case of low range communication, using a mobile gateway instead of stationary one considerably decreases network overhead. This is because the mobile gateway is closer to the centre of topology than a stationary gateway placed on the rim of the simulation area so the lengths of multi-hop paths are shorter. The mobile gateway also comes to the close proximity of a larger number of nodes which decreases the number of retries.

Figure $3 \mathrm{c}$ shows the influence of number of network nodes on SR in conditions of lowest possible stocking density ( 2 animals/ha). The differences between high and low bandwidth wireless communications are smaller than the differences between types of gateways. As it can be clearly spotted the mobile gateway performs considerably better than the stationary one. This is because the mobility of the gateway overcomes the fact that the topology splits into separated islands of connectivity. The performance in the worst case, a single stationary gateway and low bandwidth communication, is still above $90 \%$.
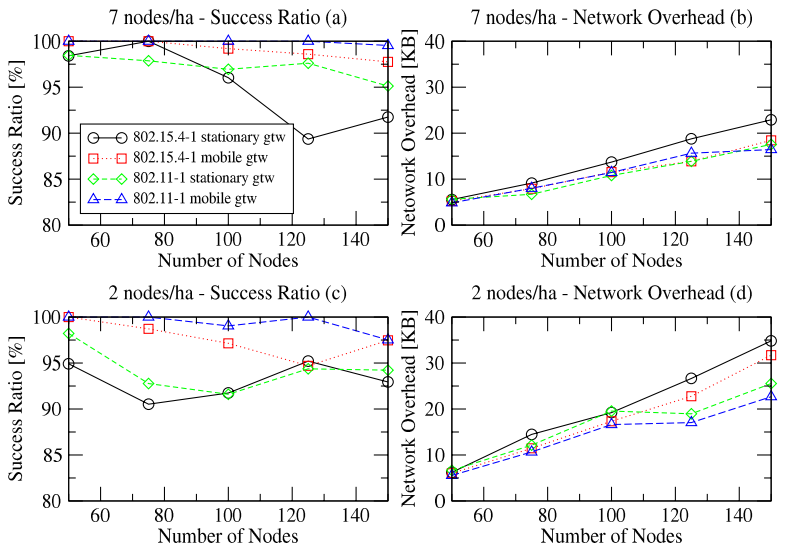

Figure 3. Simulation results

Figure $3 \mathrm{~d}$ shows the influence of number of nodes on network overhead (NO) in conditions of lowest possible stocking density. NO in case of lower bandwidth communication is higher as the sparse topology causes some bottlenecks vulnerable to congestion. Using a mobile gateway produces smaller network overhead because it is closer to the centre of the network topology and uses its mobility to come into proximity of larger number of nodes. 
To summarize, in all examined cases at least $89 \%$ of nodes succeed to send their measurements to the gateways within 2 hours, i.e. before the next chunk of data is produced. It is a very good result as in practice nodes have much more time to transfer their data. The important observation is that using low range and low bandwidth communication is sufficient to deliver data to the gateways regardless of number of nodes and stocking density. Regardless of condition using a mobile gateway considerably improves the reliability of data delivery and slightly increases the network overhead.

\section{Conclusions}

In this paper, we proposed a novel approach to detecting and querying events related to farm animals. This approach utilizes the available networking infrastructure but can also work in a fully ad hoc infrastructure-less conditions. Notifications sent to mobile phones and displayed on message boards together with ability to query the real time and historic data from home and office PCs or a mobile phone makes the stockmen always up to date with the situation on the farm. Therefore, they can take care of the animals whenever it is necessary.

\section{References}

[1] H. Williams, N. Reeves, D. Jones, and W. Morris, "The economics of lowland beef production in England, 2003 : a report on the results of a special study of lowland beef enterprises in England", Aberystwyth : The University of Wales, Farm Business Survey Unit, Aberystwyth, 2005.

[2] K. Mayer, K. Ellis, and K. Taylor, "Cattle Health Monitoring Using Wireless Sensor Networks", In Proc. of The 2nd IASTED International Conference on Communication and Computer Networks, Cambridge, Massachusetts, USA, 2004.

[3] B. Lowman, "Triple synchronisation of suckler cows", In Practice, 31 January 2003, 25 (1), pp. 38-43(6).

[4] M. Radenkovic and B. Wietrzyk, "Wireless Mobile Adhoc Sensor Networks for Very Large Scale Cattle Monitoring", In Proc. of ASWN, Berlin, Germany, 2006.

[5] C. J. C. Phillips, Principles of cattle production, CABI Publishing, Oxon, UK, 2001.

[6] W. Prichard, Cattle Handling - Leading The Way Forward, NFST Secretatiat, East Holme Farm, Maresfield, Uckfield, East Sussex, UK, 2000.

[7] A. Frost, C. P. Schofield, S. A. Beaulah, T. T. Mottram, J. A. Lines, and C. M. Wathes, "A review of livestock monitoring and the need for integrated systems", Computers and Electronics in Agriculture, 1997, 17, pp. 139-159.
[8] W. J. Eradus and M. B. Jansen, "Animal identification and monitoring", Computers and Electronics in Agriculture, 1999, 24, pp. 91-98.

[9] K. Maatje, R. M. d. Mol, and W. Rossing, "Cow status monitoring (health and oestrus) using detection sensors", Computers and Electronics in Agriculture, 1997, 16 (3), pp. 245-254(10).

[10] R. M. d. Mol, A. Keen, G. H. Kroeze, and J. M. F. H. Achten, "Description of a detection model for oestrus and diseases in dairy cattle based on time series analysis combined with a Kalman filter", Computers and Electronics in Agriculture, April 1999, 22 (2), pp. 171185(15).

[11] R. Firk, E. Stamer, W. Junge, and J. Krieter, "Automation of oestrus detection in dairy cows: a review", Livestock Production Science, July 2002, 75 (3), pp. 219-232(14).

[12] M. N. Velasco-Garcia and T. Mottram, "Biosensors in the livestock industry: an automated ovulation prediction system for dairy cows", Trends in Biotechnology, 1 November 2001, 19 (11), pp. 433433(1).

[13] R. E. Kenward, A Manual for Wildlife Radio Tagging, Academic Press, 2001.

[14] A. Mainwaring, J. Polastre, R. Szewczyk, D. Culler, and J. Anderson, "Wireless Sensor Networks for Habitat Monitoring", In Proc. of WSNA, Atlanta, USA, 2002.

[15] "Wildlife Computers", URL: http://www.wildlifecomputers.com/.

[16] "Habit Research", URL: http://www.habitresearch.com/.

[17] "Lotek Fish and Wildlife Monitoring Systems - Radio, Acustic, Satellite \& Archival Telemetry Solutions", URL: http://www.lotek.com/.

[18] Televit, "GPS collars", URL: www.televit.se.

[19] "Advanced Telemetry Systems", URL: www.atstrack.com.

[20] "Environmental Studies", URL: http://www.environmental-studies.de/.

[21] P. Zhang, C. M. Sadler, S. A. Lyon, and M. Martonosi, "Hardware Design Experiences in ZebraNet", In Proc. of SenSys, Baltimore, Maryland, USA, 2004.

[22] Z. Butler, P. Corke, R. Peterson, and D. Rus, "Dynamic Virtual fences for Controlling Cows", In Proc. of Ninth International Symposium on Experimental Robotics, Marina Mandarin Hotel, Singapore, 2004.

[23] L. Breslau, D. Estrin, K. Fall, S. Floyd, J. Heidemann, A. Helmy, P. Huang, S. McCanne, K. Varadhan, Y. Xu, and $\mathrm{H}$. Yu, "Advances in Network Simulation", IEEE Computer, 2000, 33 (5), pp. 59-67.

[24] C. J. C. Phillips, Cattle behaviour, Farming Press, Ipswich, UK, 1993.

[25] D. B. Johnson and D. A. Maltz, "Dynamic Source Routing in Ad Hoc Wireless Networks": Kluwer Academic, 1996.

[26] C. E. Perkins and E. M. Royer, "Ad-hoc On-Demand Distance Vector Routing", In Proc. of WMCSA, 1999. 